\title{
Pour une étude globale de l'iconographie des omeyyades de Syrie et d'Espagne (VIII-XIe): le cas des calendriers et des thèmes agricoles ${ }^{1}$
}

\author{
Nadia Ali \\ Université Paris IV-la Sorbonne (Paris). Université de Provence (Aix en Provence) \\ Laboratoire d'archéologie médiévale et méditerranéenne (LAMM-Aix en Provence) \\ izzayyek@yahoo.fr
}

De 639 à 750, la Syrie est dominée par le clan des Omeyyades². Chronologiquement cette période correspond à la fois à l'organisation de l'empire et à la formation de l'art islamique. Avant d'envisager les rapports possibles entre la production artistique des Omeyyades de Syrie et celle de leurs successeurs occidentaux, il est essentiel de connaître le mieux possible les monuments des Omeyyades de Syrie parce que ces constructions et leurs décors constituent un des rares moyens dont dispose l'historien pour accéder directement à l'image que les Omeyyades désiraient projeter et laisser d'eux-mêmes. Cette image que l'on peut saisir, notamment, dans les décors des résidences extra-urbaines de Qusayr 'Amra (après 711)3. Khirbat al-Mafjar (724-743) et Qasr al-Hayr al-Gharbî (728) présente l'avantage de ne pas avoir été "filtrée ou

1 Ce titre est inspiré de l'article de P. CHALMETA, "Pour une étude globale des Omeyyades", P. CANIVET (Ed.), La Syrie de Byzance à L’Islam, Damas, 1992, pp. 333-337.

2 H. KENNEDY, The Prophet and the Age of the Caliphates, Longman, 1986, pp. 82-123; G. HAWTING, The First Dynasty of Islam, Carbonsdale, 1987. Pour une vision archéologique voir D. WHITCOMB "Umayyad and Abbasid periods", B. Mc DONALD (Ed.), The Archaeology of Jordan, Sheffield, 2001 pp. 503-513; A. WALMSLEY, Early Islamic Syria, London, 2007; A. NORTHEDGE, Studies on Roman and Islamic Amman, Oxford, 1992.

3 Pour une bonne synthèse des problèmes de datation de Qusayr 'Amra, voir A. BORRUT, L'espace syrien sous les derniers Omeyyades, Leiden, 2011, pp. 418-421.

4 A propos des résidences extra-urbaines omeyyades, voir H. LAMMENS, Etudes sur le siècle des Omayyades, Beyrouth, 1930, pp. 325-350; E. HERZFELD, "Mshatta, Hira und Badiya: Die Mittellander des Islam und ihre Baukunst”, Jahrbuch der Preuzischen Kunstsammlungen, 42 (1921), pp.104-146; O. GRABAR, Ceremonial and Art at the Umayyad Court, Unpublished Ph.D Dissertation, Princeton, 1955, pp. 283-309; Ibidem, The Formation of Islamic Art, New Haven, 1987, pp.132-169; Ibidem et R. HOLOD, City in the desert, Cambridge, 1978; Ibidem, "Umayyad Palaces Reconsidered", Ars Orientalis 23 (1993), pp. 93-108; D. SCHULMBERGER, Qasr al-Hayr al-Gharbi, Paris, 1980; R. HILLENBRAND, "La Dolce Vita in Early Islamic Syria", Art History, 5 (1992), pp. 1-35; B. GEYER, "Des fermes byzantines aux palais omayyades ou l'ingenieuse mise en valeur des plaines chalcidiques", L. NORDIGUIAN (Ed.), Aux origines de l'archéologie aérienne: A. Poidebard (1878-1955), Beirut, 2000, pp.109-122; G. KING, "Settlements Patterns in Islamic Jordan: The Umayyad use of the Land" Studies on the History and Archaeology of Jordan, 4 (1992), pp. 369-375; D. GENEQUAND, "Formation et devenir du paysage architectural omeyyade: l'apport 
refaçonnée" contrairement aux textes abbassides qui offrent des Omeyyades une description souvent biaisée et partielle. Malheureusement, l'analyse des décors des "châteaux du désert" souffre de nombreux handicaps: l'absence d'information fiable sur la fonction des châteaux, l'identité des commanditaires et des artisans, et l'intention qui a présidé au choix et à la distribution des motifs dans les programmes iconographiques. On ne sait rien sur la manière dont les Omeyyades ont compris les thèmes iconographiques qu'ils ont empruntés au vocabulaire artistique de l'Antiquité tardive et aux traditions protobyzantines et sassanides. Cette lacune documentaire a conduit la plupart des analystes d'Oleg Grabar à Garth Fowden à inscrire les décors au cœur d'un faisceau de textes historiques et littéraires censés à en donner une explication d'ensemble, mais le plus souvent de détail. A y regarder de près, la plupart des interprétations que l'on considère comme acquises sont en fait le produit de spéculations iconologiques invérifiables où tout devient symbole de n'importe quoi. A ce titre le cas "du cycle princier" est particulièrement révélateur. Dans une première partie, nous montrerons que certaines parties des décors des "châteaux du désert" qui ont été interprétées par Oleg Grabar et d'autres comme la représentation de "cycles princiers" ou de la cour omeyyade représentent en fait des calendriers agricoles inspirés des almanachs de l'Antiquité tardive et du monde chrétien oriental. Comme nous le verrons ces calendriers omeyyades sont fréquemment représentés en collocation avec la figure du prince ou du calife. Ils forment avec l'image du souverain une structure thématique récurrente que l'on retrouve dans les trois résidences (Qusayr 'Amra, Khirbat al-Mafjar et Qasr al-Hayr al-Gharbî) et dans des techniques décoratives variées (peinture, stuc). Cette constante iconographique semble participer d'une volonté du pouvoir omeyyade de se présenter comme un pouvoir nourricier, garant de la fertilité tout au long de l'année sur le modèle d'ailleurs de la propagande romaine ou byzantine (Barakat, ni'mat, fadl, surur et Felicitas). Dans la seconde partie, on se tournera vers les ivoires produits en al-Andalus au Xe siècle en se questionnant sur les conséquences de la découverte des calendriers syriens sur notre compréhension de certains aspects iconographiques des ivoires. Il s'agira principalement de repérer les thèmes agricoles et calendricaux sur les ivoires en exploitant conjointement la documentation visuelle et les sources textuelles, en particulier le Calendrier de Cordoue (961). Enfin dans une troisième partie, on s'interrogera sur les facteurs qui ont pu motiver l'utilisation de ces thèmes "inhabituels" dans la mise en scène du pouvoir en al-Andalus. Doit-on y voir un écho lointain de la propagande visuelle de leurs ancêtres syriens ou le produit de la rivalité mimétique qui oppose le califat des Omeyyades d'Espagne à celui des Abbassides de Bagdad et des Fatimides d'Egypte?

\footnotetext{
de l'archéologie", A. BORRUT et P. COBB, (Eds.), Umayyad Legacies, Leiden, 2010, pp. 417-473; Ibídem, "Umayyad Castles: The Shift from Late Antique military architecture to early Islamic palatial buildings", $\mathrm{H}$. KENNEDY (Ed.), Military Architecture in Greater Syria, Leiden, 2006, pp. 10-22.
} 


\section{L'ICONOGRAPHIE ROYALE DES OMEYYADES DU BILÂD AL-SHÂM REVISITÉE}

\subsection{LE SCHÉMA NARRATIF TRADITIONNEL ET SON PRINCIPAL RESSORT}

L'essentiel de la documentation sur l'iconographie royale des Omeyyades de Syrie provient des décors des établissements ruraux du Bilâd al-Shâm mieux connus sous le nom de "châteaux du désert". Trois d'entre eux présentent des programmes iconographiques particulièrement riches: Qusayr 'Amra (peintures murales et mosaïques), Khirbat al-Mafjar (stucs, mosaïques et peintures) et Qasr al-Hayr al-Gharbî (stucs et peintures). Il est souvent question de "cycles princiers" ou de "passe-temps princiers" dans la littérature archéologique et historique consacrée aux décors de ces établissements aristocratiques. Certes, tous les décors palatiaux omeyyades ne concernent pas "le cycle princier" et n'ont pas été interprétés comme tel ${ }^{5}$, mais que ce soit pour Qusayr 'Amra (Jordanie, 730) ${ }^{6}$, Khirbat al-Mafjar (Palestine, 743) 7 et Qasr al-Hayr al-Gharbî (Syrie, 728) ${ }^{8}$, l'historiographie a massivement utilisé les cycles princiers comme grille de lecture de l'iconographie royale omeyyade'. C'est Oleg Grabar qui dès 1955 formule cette hypothèse d'une prédominance de thèmes consacrés aux passe-temps princiers et ce en se fondant sur deux types d'images: d'un côté les images qui représentent indiscutablement des passe-temps royaux: (musiciens, chasse royale, danseuses nues) et de l'autre, de larges ensembles des programmes

\footnotetext{
5 Je pense notamment au zodiaque du caldarium, aux scènes montrant la construction d'un édifice à Qusayr 'Amra ou aux représentations de Gê à Qasr al-Hayr al-Gharbî.

6 Pour une étude de l'architecture de Qusayr 'Amra, voir K. A. C. CRESWELL, Early Muslim Architecture, Oxford, 1932, pp. 255-272; J. SAUVAGET, "Remarques sur les monuments omeyyades de Syrie", Journal Asiatique, 231 (1939), pp. 13-16; M. ALMAGRO et alii, Qusayr Amra, Madrid, 1975, pp. 25-48; G.BISHEH, T. MORIN et C.VIBERT-GUIGUE, "Rapport d'activités à Qusayr Amra", Annual of the Department of Antiquities of Jordan, 41 (1997), pp. 375-393; G. FOWDEN, Qusayr 'Amra, Berkeley, 2004, pp. 31-57; T. MORIN et C. VIBERT-GUIGUE, "Une structure d'accueil des visiteurs à l'entrée de Qusayr Amra", Annual of the Department of Antiquities of Jordan, 44 (2000), pp. 581-591.

7 R. HAMILTON, Khirbat al-Mafjar. Oxford, 1954.

8 D. SCHLUMBERGER, op. cit., 1980.

9 O. GRABAR, op. cit., 1955, pp. 1-2 où on peut lire: "The figurative remains from the three palaces of Qusayr Amra, Khirbat al-Mafjar and Qasr al-Hayr al-Gharbi, although different in style, show iconographic similarities. The main theme is the king surrounded by musicians, dancers, athletes and hunting scenes", voir aussi les pages 14, 192-233. Voir aussi, Ibidem, La formation de l'art islamique, Paris, 1986, pp. 232-233 où on peut lire "le cycle princier est présent dans tous les palais omeyyades (...) A part le cycle princier et ses variantes, il est presque impossible de distinguer des catégories iconographiques claires"; Ibidem, op. cit., 1993, p. 96; R. HILLENBRAND, op. cit, 1992, pp. 1-20, en particulier les p. 9-10; C.VIBERTGUIGUE et G.BISHEH, Les peintures de Qusayr 'Amra, Beyrouth, 2007, pp. 8; M. ALMAGRO et alii, op. cit., 1975, pp. 56; E. BAER, The Human Figure in Islamic Art, Costa Mesa, 2004, pp. 5-6; O.GRABAR, R. ETTINGHAUSEN, et M. JENKINS-MADINA, The Art and Architecture of Islam, New Haven, 2001, pp. 45-48; M. VAN LOHUIZEN-MULDER, "Frescoes in the Muslim Residence and bath-house of Qusayr "Amra", BABESCH, Annual Papers on Mediterranean Archaeology, 73 (1998), pp. 125-151; pp. 128-129; G. FOWDEN, op.cit., 2004, pp. 60-61, 78. Dans cette monographie consacrée en 2004 à Qusayr Amra, G. Fowden reprend sans la nommer l'idée des cycles princiers. Il écrit: "Abundance, relaxation and eroticism; music making, singing and dancing, the showing of fine clothing and jewelery by women, all these things were, and long remained, part of bath-house culture (...) Yet, as with all pleasure they took their special intense taste from contrast with exertion and risk, whether in hunt (...) or in war, ever part of Umayyad life".
} 
tels que les peintures de la travée centrale de Qusayr 'Amra, et les figures sculptés en stuc du tambour du porche de Khirbat al-Mafjar et celles de la façade sur cour de Qasr al-Hayr al-Gharbî. En exploitant conjointement l'iconographie et les textes de l'époque abbasside, $\mathrm{O}$. Grabar interprète ces ensembles comme une mise en scène du pouvoir omeyyade inspiré du cérémonial sassanide où les princes se montraient entourés de leur cour et de personnages censés les divertir (porteurs d'offrandes, danseuses, musiciens, échansons, acrobates).

Les textes utilisés par O.Grabar sont le plus souvent des sources rédigées non pas dans la Syrie omeyyade, mais dans l'Iraq abbasside des IX-Xe siècles (le Kitâb al-Aghânî d'al-Isfahan $\hat{1}^{10}$ ou les chroniques des historiens tels que Tabarî, Mas'udî). Ces textes qui évoquent le plus souvent les déviances des Omeyyades (introduction de la royauté dans l'Islam, paresse, excentricité des califes, goût pour la boisson, les femmes et la musique) ont considérablement influencé notre vision de la première dynastie de l'Islam et de sa production artistique. En effet, pour beaucoup de chercheurs, l'opulence des décors des établissements omeyyades semble confirmer les descriptions fournies par les sources textuelles arabes sur le caractère libertin et déviant des souverains omeyyades. De même que jusqu'à récemment, la situation de ces résidences à l'extérieur des villes était interprétée comme la preuve d'une volonté omeyyade de jouir des plaisirs de la vie de cour loin des regards ${ }^{11}$. Si les textes abbassides ont conditionné l'hypothèse du "cycle princier", ils ne la valident pas pour autant. D'abord parce qu'on sait grâce aux progrès de l'archéologie protobyzantine ${ }^{12}$ et islamique dans le Bilâd al-Shâm que les qusur omeyyades avaient de multiples fonctions. Plusieurs hypothèses ont été émises: résidence saisonnière, nœud caravanier, pavillon de chasse, lieu de réunion entre les princes omeyyades et les tribus arabes qui nomadisaient dans la région. Plus récemment la recherche archéologique à partir de l'analyse de la topographie a permis de mettre en évidence le réseau de ces châteaux et un maillage très serré conduisant à l'hypothèse selon laquelle ces établissements servaient de bases logistiques à l'exercice mobile du pouvoir $^{13}$. Ensuite, même s'il est vrai que certains princes omeyyades semblent avoir mené une vie particulièrement excentrique (al-Walid II), la vision d'une dynastie décadente offerte par les sources abbassides a été remise en question par les travaux historiographiques récents d'Antoine Borrut ${ }^{14}$ et de Fred M. Donner ${ }^{15}$. Cette révision radicale n'a jusqu'ici pas eu de conséquence sur notre perception des décors palatiaux omeyyades. La plupart des travaux, y compris les plus récents continuent de voir dans

10 H. KILPATRICK, "Umar ibn 'Abd al-'Aziz, al-Walid ibn Yazid and their Kin: Images of the Umayyads in the Kitâb al-Aghâni”, A. BORRUT et P. COBB (Eds.), Umayyad Legacies, Leiden, 2010, pp. 63-89.

11 R. HILLENBRAND, op. cit., 1992, pp.1-20.

12 J. P. SODINI, “L'apport de l'archéologie", Dumbarton Oaks Papers, 54 (2000), pp.139-184.

13 Sur la fonction des établissements ruraux omeyyades voir D. GENEQUAND, Les élites omeyyades de Palmyrène: contribution à l'étude des aspects fonctionnels et économiques des établissements aristocratiques du Bilâd al-Shâm, Thèse de doctorat non publiée, Paris, 2010, pp. 245-324; G. KING, op. cit., 1992, pp. 369375; A. BORRUT, Entre mémoire et pouvoir. L'espace syrien sous les derniers Omeyyades, Leiden, 2010, pp. 383-466.

14 Ibídem, pp. 166-203.

15 F. DONNER, "Umayyad Efforts at Legitimation", A. BORRUT et P. COBB (Eds.), Umayyad Legacies, pp. 188. 
l'iconographie palatiale des Omeyyades le reflet des pratiques "déviantes" des élites Omeyyades ${ }^{16}$. Pourtant, on va voir que loin d'être une donnée immédiate, l'existence de cycles consacrés à la représentation des divertissements princiers, qui pour être la mieux accréditée, n'est cependant pas la seule possible et surtout s'accommode au plus mal des données disponibles dans les images elles-mêmes.

\subsection{IDENTIFICATION DES CALENDRIERS AGRICOLES DANS LES DÉCORS OMEYYADES}

Nous allons dans les lignes qui suivent mettre à l'épreuve l'hypothèse des "cycles princiers" en réexaminant les décors sous l'angle de vue bien particulier de leur fabrication. Le cas de Qusayr 'Amra, un bain doublé d'une salle d'audience et construit dans la Balqaa vers 730 par al-Walid II (ou Sulayman) servira d'exemple clef. Etant donné les nombreux problèmes de lecture que posent ce décor et les nombreux repeints ${ }^{17}$, plusieurs éléments d'identification doivent être conjugués pour identifier "correctement" les thèmes iconographiques.

1) Le premier élément de reconnaissance correspond à ce qu'Otto Pacht appelait "l'occasion formelle"18. Ce terme désigne deux choses: premièrement le rapport de dépendance entre le contexte architectural, les schémas organisateurs appliqués au décor et les choix iconographiques; et deuxièmement une procédure analytique qui consiste à ramener le décor à son point de départ, c'est à dire ses surfaces murales vides. L'objectif étant à la fois de définir les contraintes de l'architecture sur l'iconographie, mais aussi de commencer à pénétrer l'univers technique et mental des artisans du Bilâd al-Shâm. Bien que cela ne soit pas sa fonction première, l'application du principe de l'occasion formelle à l'analyse du décor de Qusayr 'Amra va nous permettre de proposer une autre identification des thèmes. Dans la formulation de son hypothèse des cycles princiers à Qusayr 'Amra, $\mathrm{O}$. Grabar se fonde essentiellement sur le programme peint de la travée centrale qu'il interprète comme la représentation du calife et de sa cour (fig.1 et 3). C'est donc cette partie du programme, et en particulier le décor du plafond central qui seront réexaminés ici. La surface décorée du plafond central est organisée par un quadrillage. Le quadrillage employé présente quelques affinités avec les décors de plafonds peints du nord de la Jordanie (Loukanios, IIIe siècle) $)^{19}$ et surtout avec les mosaïques du Proche-Orient. Les églises de Jordanie et leurs mosaïques comptent en effet de nombreuses occurrences de l'usage du quadrillage comme canevas appliqué au décor. L'église des Apôtres à Madaba

16 M. MILWRIGHT, "Archaeology and Material Culture", C. ROBINSON (Ed.), The New Cambridge History of Islam, Cambridge, 2010, p. 667. On peut lire dans le chapitre consacré à la culture matérielle omeyyade: "The opulent decoration encountered on sites such as Khirbat al-Mafjar and Qusayr Amra offers intriguing insights into the culture of Umayyad princely pleasure known otherwise from textual sources".

17 Notons ici que les peintures de Qusayr 'Amra font actuellement l'objet de restauration. Ce qui implique que certains éléments de notre analyse pourrait être révisés dans le futur.

18 O. PACHT, Questions de méthode en histoire de l'art, Paris, 1994, pp. 48-52.

19 A. BARBET, Les peintures des nécropoles romaines d'Abila et du nord de la Jordanie, Beyrouth, 19941998, p. 19. 


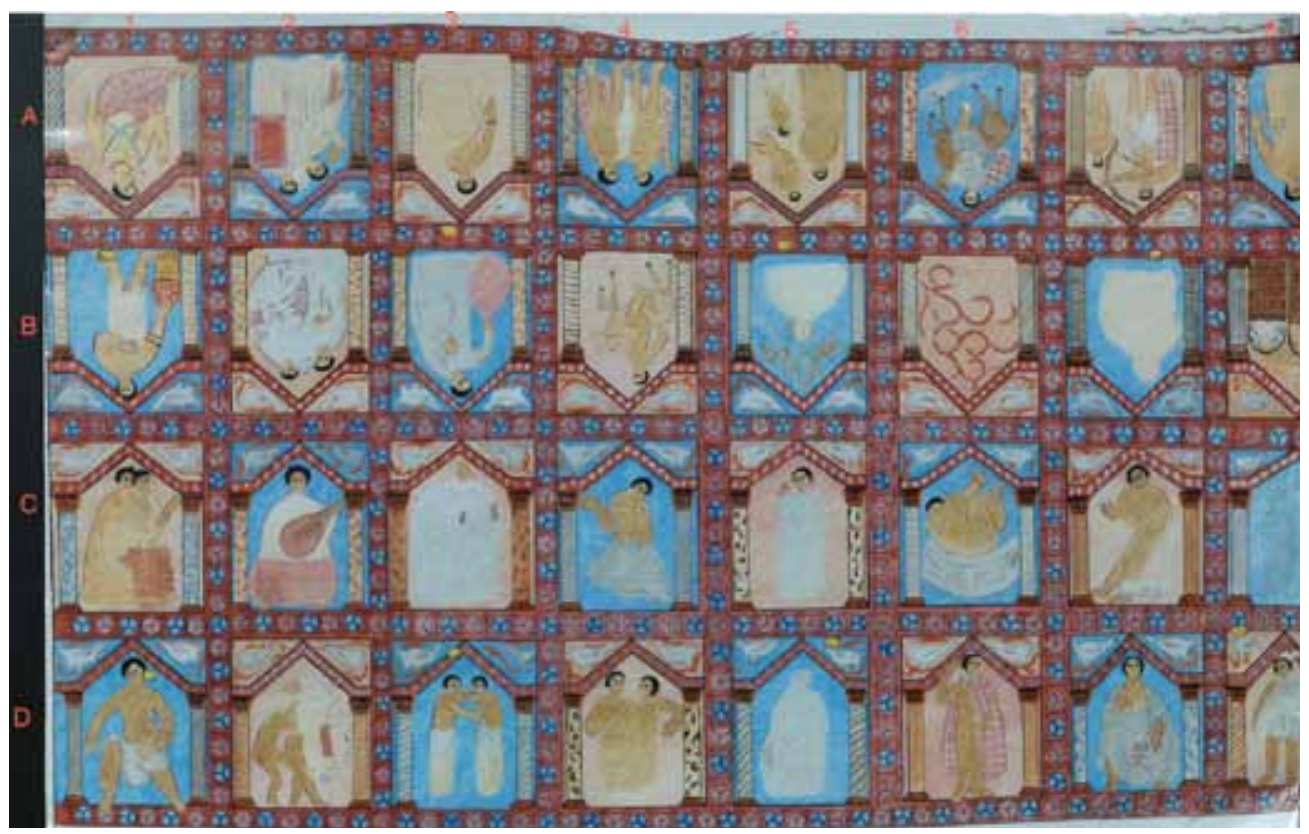

Fig. 1. Le décor du plafond de la travée centrale de Qusayr 'Amra : un calendrier monumental [dessin d'après C.VIBERT-GUIGUE et G.BISHEH, Les peintures de Qusayr 'Amra, Beyrouth, 2007, pl. 86].

(578), par exemple, présente des pavements de mosaïques quadrillées ${ }^{20}$. C'est aussi le cas dans les pavements des églises de Saint Pierre (fin du VIe siècle) et de Saint Elias, Marie et Soreg à Jerash (VIe siècle) ${ }^{21}$. L'objectif des exemples précités n'est pas de dresser une sorte de filiation généalogique entre le décor omeyyade et ses ancêtres supposés. Il s'agit d'insister sur un point simple de notre argument: le découpage appliqué au plafond de Qusayr 'Amra pourrait correspondre à une habitude de métier et a été conditionné par la nature architecturale de l'espace à décorer (un plafond voûté). Il s'agit ensuite de déterminer dans quelle mesure le découpage a exercé à son tour une force de suggestion sur le choix des formes et des sujets qui s'y insèrent. On part ici de l'hypothèse selon laquelle suivant la configuration ou le format géométrique des champs découpés, les artisans auront recours à un certains type de schèmes ou de thèmes. Dit autrement, on ne décore pas la surface triangulaire d'un écoinçon comme on décorerait la surface demi-circulaire d'une lunette. Pour déterminer si le contexte architectural et le découpage sont susceptibles de déclencher des attentes de sujets spécifiques, il faut faire un sondage dans les décors préislamiques qui présentent des

20 M. PICIRILLO, The Mosaics of Jordan, Amman, 1993, p. 95

21 G. AKERSTROM, The Calendar and Hunting Mosaics of the villa of the Falconer in Argos, Stockholm, 1974, fig. 82.3 . 


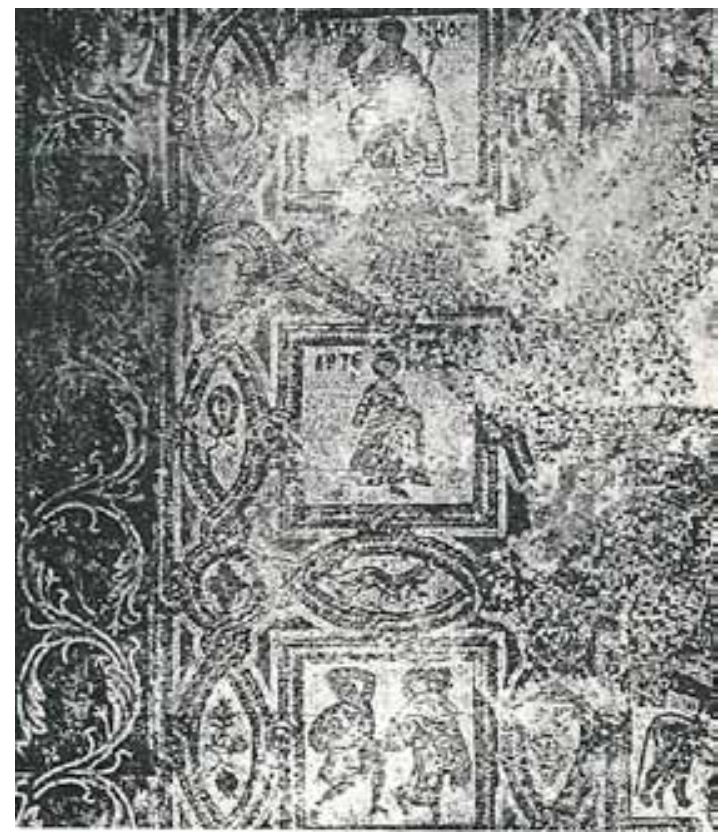

Fig. 2. Le calendrier de l'église Saint Elias, fragments de mosaïque montrant le mois de Janvier, Jerash, VIe siècle [d'après G. ACKERSTROM, The calendar and hunting mosaics of the villa of the falconer in Argos, Stockholm 1974, fig 82.3].

affinités organisationnelles avec le plafond omeyyade. L'objectif ici est de donner une idée de quelques uns des thèmes les plus fréquemment associés au quadrillage dans les décors du Proche-Orient à la veille de l'Islam. Un premier sondage révèle la fréquente occurrence du thème du calendrier illustré dans les canevas quadrillés. Par exemple, l'église de Jerash était ornée d'un grand pavement quadrillé où étaient représentés les Saisons et les mois de l'année identifiés par des inscriptions. Les iconoclastes ayant détruit les figures de ce calendrier, il est impossible aujourd'hui de le décrire. Un autre pavement quadrillé et représentant un calendrier a été également mis au jour à Jérash dans l'église de Saint Elias, Marie et Soreg (VIe siècle). Seules quelques personnifications de mois peuvent être identifiées. Le mois de janvier sous les traits d'un consul levant la mappa et tenant un sceptre, faisant ainsi allusion à la désignation du consul en janvier (vota) et au lancement des jeux (fig. 2). Ces calendriers de Jerash suggèrent un lien structurel entre le canevas défini par un quadrillage et le thème du calendrier. On pourrait d'ailleurs renforcer cette hypothèse par d'autres mosaïques de l'Antiquité tardive telles que celle de Djem (Tunisie, IIIe siècle) ${ }^{22}$,

22 K. DUNBABIN, Mosaics of Roman North Africa: studies in iconography and patronage, Oxford, 1978, fig. 20 . 
d'Argos ou de Tégée (Grèce, Ve siècle) ${ }^{23}$ qui toutes utilisent un quadrillage pour mettre en image la représentation des mois de l'année. Notons aussi que le thème du calendrier agricole est très courant dans l'art de la fin de l'Antiquité, y compris au Proche Orient comme l'indiquent les mosaïques de la Villa d'Awza'i ou celles de l'église de Qabr-Hiram (Liban, VIe siècle). Aussi, si on se fonde sur la structure formelle du plafond omeyyade actualisée par un quadrillage et sur sa situation dans la travée centrale, on peut à la suite des exemples cités émettre l'hypothèse d'un calendrier.

2) Le second élément de reconnaissance correspond à ce que ce nous pourrions appeler "des structures thématiques ou collocation de sujets": c'est à dire des thèmes que les artisans de la région avaient pour habitude de représenter ensemble. Les thèmes qui fonctionnent traditionnellement avec les calendriers sont multiples mais les plus récurrents sont les personnifications des Saisons et les allégories de Ge ou de l'Océan. Or ces sujets se retrouvent dans le décor de l'alcôve qui se trouve dans le prolongement de la travée centrale où on peut voir des figures inspirées des Saisons sous des arcs et surplombées de bustes de Gê, la déesse de la terre (fig. 3). L'ensemble encadre l'image d'un prince omeyyade trônant au dessus d'un paysage nilotique. A ce stade, il n'est pas inutile de rappeler que les thèmes comme Gê, les Saisons et les calendriers étaient utilisés comme insigne de pouvoir dans la propagande de l'art de l'Antiquité tardive. De ce fait, la combinaison qui montre un prince trônant sur l'eau, les Saisons, Ge et le calendrier présente une certaine cohérence sémantique.

3) Le dernier élément d'identification est l'iconographie (fig. 1). Bien qu'il soit difficile de retrouver un sens de lecture chronologique dans ce calendrier, il est possible d'y reconnaitre plusieurs figures typiques des calendriers : le mois de janvier représenté en consul qui jette la mappa pour annoncer le début des festivités du nouvel an (D6-D7); la personnification du mois d' octobre en chasseur oiseleur (B3); des scènes en rapport avec les travaux agricoles propres à chaque mois comme les semailles, les paniers garnis représentant les produits récoltés (B1) ou des scènes de vendange associées au mois de septembre (D2-D3); plusieurs signes du zodiaque et notamment les gémeaux (A4) ${ }^{24}$ et le sagittaire (A7). Le calendrier de Qusayr 'Amra comporte aussi des représentations de musiciens et surtout l'image d'un cavalier qui semble tenir un faucon (B4). Cette image pourrait être rapprochée des reliefs récemment mis au jour à Qasr al-Hayr al-Gharbî. La présence de fauconnier dans l'iconographie d'un calendrier peut être en partie expliquée par une tradition artistique puisque les fauconniers apparaissent aussi dans le calendrier de Jerash et celui d'Argos.

Il ressort que l'analyse iconographique, le contexte architectural et le canevas appliqué au décor confirment l'hypothèse d'un calendrier dans la travée centrale de Qusayr 'Amra. C'est donc à la lumière de cette nouvelle identification qu'il convient de relire les décors en stuc du porche de Khirbat al-Mafjar et celui de Qasr al-Hayr al-Gharbî.

23 G. AKERSTROM, op. cit., 1974, p. 41.

24 Le signe des gémeaux avait déjà été identifié par A. Almagro. M. ALMAGRO et alii, op. cit., 1975. 


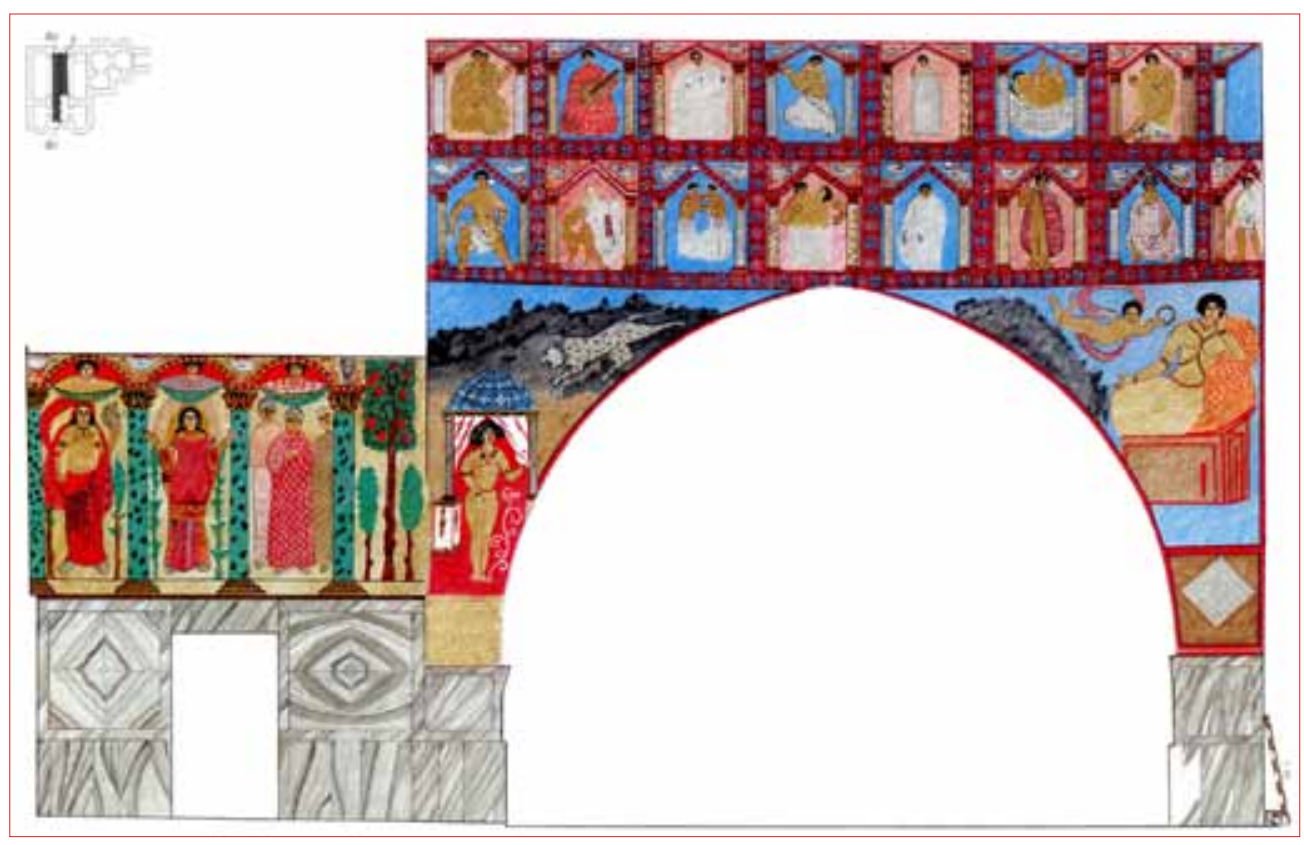

Fig. 3. Ensemble iconographique de la travée centrale de Qusayr 'Amra (Gê, Saisons et calendrier). [d'après la reconstitution de C.VIBERT-GUIGUE et G.BISHEH, Les peintures de Qusayr 'Amra, Beyrouth, 2007, pl. 122b].

Khirbat al-Mafjar est un palais doublé d'une salle d'audience et de bains construits près de Jericho entre 724 et 743 et attribué tantôt au calife al-Hishâm tantôt à al-Walid II. L'ensemble iconographique qui décore le porche de la grande salle de réception a été interprété comme la représentation du calife et de sa cour. D'autres y ont décelé des allusions à la figure de Salomon ${ }^{25}$. Pourtant plusieurs indices suggèrent qu'on pourrait là aussi avoir à faire à un calendrier monumental. Premièrement, le décor orne le tambour d'une coupole. Or, il existe une longue tradition décorative attestée depuis l'Antiquité et qui consiste à décorer les coupoles de thèmes cosmiques ou calendricaux ${ }^{26}$. Le réexamen des figures omeyyades va également dans ce sens. La plupart des représentations féminines portant des paniers de fleurs ou de fruits présentent de nombreuses affinités avec les Horae ou les Saisons que l'on peut voir sur plusieurs calendriers (Manuscrit du Vatican) ${ }^{27}$. L'image d'un guerrier en armure

25 P. SOUCEK, “Salomon's trone, Salomon's bath”, Ars Orientalis, 23 (1993), pp. 109-134. A. BORRUT, op.cit., 2010, pp. 217-224.

26 K. LEHMAN, "The Dome of Heaven", The Art Bulletin, 27 (1945), pp. 1-27. Voir aussi D. LEVI, "The Allegories of the Months in Classical Art”, The Art Bulletin, 23 (1941), pp. 251-292 en particulier p. 281.

27 Miniature du manuscrit de Ptolémée daté du IXe siècle (copie d'un original du III $^{\mathrm{e}}$ siècle), Bibliothèque du Vatican: MS GR 1291. Pour une illustration voir D. LEVI, op.cit., 1941, fig. 3. 
et tenant à ses pieds un bouclier rappelle les représentations de Mars visibles sur plusieurs manuscrits byzantins et surtout sur les mosaïques de Beisan-Scytholopolis (Israël, VIe siècle) ${ }^{28}$. Enfin, la figure montrant un jeune homme tenant un agneau peut être rapprochée du type iconographique utilisé pour représenter le mois d'avril dans les calendriers de Beisan-Scythopolis ou de Carthage ${ }^{29}$. Comme à Qusayr 'Amra, le calendrier fonctionne en collocation avec l'image d'un souverain qui se trouve sur la façade du porche. C'est aussi le cas à Qasr al-Hayr al-Gharbî, un autre établissement rural construit en 728 par al-Hishâm dans la Palmyrène. Le décor en stuc qui se trouve sur la façade intérieure de la cour présente une série de figures inscrites sous des arcs dont certaines évoquent clairement des travaux saisonniers et des allégories de mois: octobre portant des oiseaux dans le creux de ses bras, février voilé, et mars coiffé d'un bonnet phrygien.

Il ressort de ce survol de l'iconographie palatiale omeyyade: 1) la présence récurrente de calendriers agricoles composés de scènes rustiques, d'allégories des mois ou de personnifications des saisons. 2) Malgré les différences entre ces trois calendriers tant sur les plans technique que morphologique, on a remarqué qu'ils étaient systématiquement combinés à l'image du souverain, ce qui suggère une fonction comparable dans la propagande visuelle des Omeyyades. A ce stade, il n'est pas inutile de rappeler que dans l'art de l'Antiquité tardive les thèmes calendricaux et agricoles servaient la propagande romaine et byzantine en particulier pour représenter la notion Felicitas, ou l'idée d'un souverain capable de fournir l'abondance à ses sujets et ce tout au long de l'année. Ces calendriers sont ensuite récupérés dans l'imagerie chrétienne et juive du Proche-Orient où ils servent le plus souvent à symboliser les bienfaits de la nature accordés par Dieu. 3) La présence répétée de ces calendriers chez les Omeyyades indique une certaine conscience des significations préexistantes et une sorte manipulation de l'iconographie préexistante à leur avantage. Cette iconographie royale révisée apparaît désormais non plus comme une preuve des déviances omeyyades, mais comme un révélateur de l'image du "souverain nourricier, garant de la fertilité" aux premiers temps de l'Islam. Certaines sources littéraires confirment cette fonction nourricière du calife et notamment la poésie arabe où le souverain est comparé à un fleuve, à la pluie, ou à celui qui rend la terre fertile ${ }^{30}$. Cette insistance visuelle et poétique sur la fonction nourricière des califes pourrait s'expliquer par ce que Dumezil appelait "la sympathie entre bonne guidance et abondance agricole", "entre programme de paix et de vertu et prospérité". Rappelons que les califes omeyyades sont décrits comme les califes de Dieu (Khalifa Allah), choisis et guidés par Dieu (les termes mu'ayyad, muzaffar fréquemment employés dans les panégyriques omeyyades indiquent une intervention divine). Signalons enfin que ce lien entre les Omeyyades et l'eau/l'abondance s'est exprimé dans d'autres domaines.

28 Ibidem, fig. 12.

29 Ibídem, fig. 6.

30 AL-AKHTAL, Diwan, P.A. SALHANI (Ed.), Beyrouth, 1891-1925, poèmes nº 25, v.21;34, v. $34 ; 31$, v. $31 ; 11$, v. $39 ; 45$, v. 22 . Voir aussi les poèmes, $\mathrm{n}^{\circ} 7,11,13,18,25,34,37,43,44,45,57,58,75$, 97 . Pour une étude récente des panégyriques d'al-Akhtal, voir M. BAKKOUCH, Un aspect de la poésie d'al-Akhtal: le panégyrique, thèse de Doctorat non publiée, Aix en Provence, 2001, pp. 283- 314. 
Comme l'a montré Gulru Necipolglu, les décors végétaux foisonnants des mosaïques de la Mosquée de Damas et du Dôme du Rocher pourraient participer d'une même volonté omeyyade de se présenter comme les nouveaux garants de l'abondance. Dans les panégyriques les califes marwanides souvent décrits comme ceux vers qui on prie pour obtenir la pluie et on sait que les califes omeyyades de Syrie pratiquaient et dirigeaient l'istisqa, (la prière pour la pluie) ${ }^{31}$. Par ailleurs, comme l'a montré Denis Genequand, les inscriptions notamment celles qui se trouvent sur des ouvrages hydrauliques (barrage inscrit de Sadd Saysid construit par Mu'awiya, la citerne inscrite de Rimat al-Hazim ordonnée par Hishâm) et les textes abbassides attestent de l'implication du pouvoir omeyyade dans la construction d'ouvrages hydrauliques et le développement de la culture des terres ${ }^{32}$.

Cette représentation d'un pouvoir omeyyade garant de la fertilité apparaît en retour comme une preuve de leur légitimité. Aussi la mise en scène d'un pouvoir omeyyade nourricier dépasse le cadre des images "des châteaux du désert" et s'intègre dans un programme global de propagande qui a pu s'exprimer dans les décors religieux, la poésie, l'épigraphie et ce que D. Genequand appelle "les politiques de l'eau".

\section{LES THÈMES AGRICOLES ET CALENDRICAUX DANS LES IVOIRES D'AL-ANDALUS (X-XIE SIÈCLES)}

Cette forme de représentation du pouvoir à travers la figuration de thèmes agricoles et calendricaux dans des décors architecturaux semble ne pas avoir été poursuivie dans l'architecture palatiale des Omeyyades d'Espagne (755-1031). On peut se demander pourquoi les califes omeyyades d'Al-Andalus, bien que réclamant le mirât de leurs ancêtres du Bilâd al-Shâm, n'utilisent pas la décoration figurée, ni les allégories dans les décors de leurs résidences, palais, al-munyas. Il existe pourtant des affinités architecturales nombreuses entre par exemple Qusayr "Amra et "le Salon Riche" de Madinat al-Zahra destiné aux réceptions officielles du calife al-Nasir ("Abd al-Rahman III). Ce salon a une structure basilicale à trois travées et des bains annexes comparables à Qusayr 'Amra. Pourtant le décor sculpté très abondant est essentiellement végétal, géométrique et épigraphique. Le type d'iconographie royale utilisé par les élites omeyyades de Syrie s'est-il interrompu ou a t-il été poursuivi sur d'autres supports comme les objets en ivoires plus tardifs d'al-Andalus?

\subsection{BRÈVE PRÉSENTATION DES OBJETS}

On connait une trentaine d'objets en ivoire produits en Al-Andalus entre 950-1050. Ces objets (boîte ou pyxide) servaient le plus souvent de contenant à des matières

\footnotetext{
31 G. NECIPOGLU, “The Dome of the Rock as Palimpsest:'Abd al-Malik Grand Narrative and Sultan Suleyman's gloss", Muqarnas, 25 (2008), pp. 54-56.

32 D. GENEQUAND, "Economie de production, affirmation du pouvoir et dolce vita: aspects de la politique de l'eau sous les Omeyyades du BIlad al-Sham", M. AL-DBIYAT et M. MOUTON (Eds.), Stratégies d'acquisition de l'eau et société au Moyen-Orient depuis l'Antiquité, Beyrouth, 2009, pp. 157-177.
} 
précieuses (parfums, cosmétique) ou à des insignes du pouvoir (régalia). Ils sont issus pour la plupart des ateliers de Madinat al-Zahra, donc on peut supposer que la commande de ces objets relevait d'une prérogative califale. Ces objets précieux étaient d'ailleurs destinés à l'entourage de la cour (officiels comme le sahib al-shurta, Zyad b. Aflah, prince: al-Mughira, fille du calife Abd al-Rahman III ou la favorite d'al-Hakam, Subh,). Ces caractéristiques font de ces ivoires un moyen d'accéder au monde privé et aux aspirations des élites omeyyades d'al-Andalus. La plupart des ivoires présentent un décor épigraphié en arabe qui contient des formules de "bonne fortune" à l'attention du destinataire du cadeau, le nom du destinataire et de l'artiste (Khalaf, Misbah, Faraj), le lieu et l'année. Quant au répertoire iconographique déployé, il comprend des motifs végétaux souvent couvrants, des scènes dites de cour (figure trônante, musiciens, lutteurs), des scènes de chasse (fauconniers/lion contre taureau) et de combat. Certains de ces ivoires et notamment la boîte de Pampelune ${ }^{33}$ et la pyxide d'al-Mughira ${ }^{34}$ ont fait l'objet d'interprétations qui y ont vu des allusions au contexte politique de l'Espagne musulmane à la fin du Xe siècle. Mon intention n'est pas de revenir sur les diverses interprétations politiques proposées par S. Makariou, F. Prado-Vilar ou Renata Holod, mais de déterminer si les élites ont eu recours ou pas à des thèmes calendricaux et agricoles pour mettre en image leur pouvoir. Mon attention va se porter principalement sur 5 objets bien connus: la pyxide d'al-Mughira (968), la pyxide de Zyad ibn Aflah (969) la boîte de Pampelune (1004-1005), la pyxide de Sayf al-Dawla (1004-1008), et une boîte anonyme datée du début du XI siècle.

\subsection{REPÉRAGE DES THÈMES CALENDRICAUX ET AGRICOLES}

a) Cueillette et entretien des arbres: ce thème est d'abord visible sur l'un des médaillons de la pyxide d'al-Mughira où on voit deux cavaliers de part et d'autre d'un dattier cueillant les fruits de l'arbre (fig. 4). Il est assez rare de retrouver des dattiers dans l'iconographie médiévale des calendriers. En revanche les scènes de cueillette et d'entretien des cultures y sont fréquentes (à une différence près les cultivateurs ne

33 En 976, la mort d'al-Hakam et la fin Xe siècle marquent une dissolution du pouvoir califal et la montée en puissance des chambellans (ministre Ibn Abi Amir/al-Mansur) et son fils (Abd al-Malik). Ce dernier commande en 1005 deux ivoires dont celui de Pampelune. Sa représentation en figure trônante et l'inscription qui reprend les formules utilisées par les califes témoigneraient de ses aspirations au pouvoir.

34 Plusieurs lectures ont été proposées pour la pyxide d'al-Mughira. Prado Vilar et Renata Holod l'ont interprétée comme un cadeau offert par Ibn Abi 'Amir (le futur al-Mansur) à un des fils d'Abd al-Rahman III, al Mughira pour tempérer et ironiser sur ses éventuelles ambitions politiques. F. PRADO VILAR. "Circular Vision of fertility and punishment: caliphal ivory caskets from al-Andalus", Muqarnas, 14 (1997), pp. 19-41; R. HOLOD "Ivories", J. DODDS (Ed.), al-Andalus, The art of Islamic Spain, New-York, 1992, pp. 190206. Plus récemment Sophie Makariou y a vu un cadeau commandité par trois personnages possibles (Durri al-Saghir, Jawdar al-Hakami ou Fa'iq al-Mustansiri al-Nizami) et destiné à al-Mughira en hommage à sa maturité. Elle décèle par ailleurs dans l'iconographie des allusions à la nostalgie omeyyade pour la Syrie (la représentation du palmier), une mise en scène de la lutte pour le pouvoir (à travers les scènes dites secondaires et montrant des lutteurs, des chiens qui mordent des hommes, un âne attaqué par des chiens), et une allusion au fondateur de l'Andalus omeyyade à travers le symbolisme des faucons. S. MAKARIOU, "The al-Mughira Pyxis and Spanish Ivories” A. BORRUT et P. COBB (Eds.), Umayyad Legacies, Leiden, 2010, pp. 313-335. 


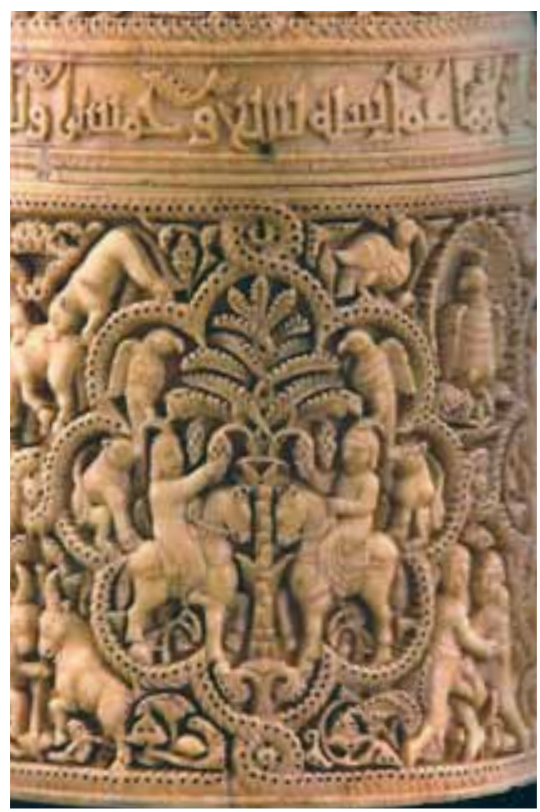

Fig. 4. Pyxide d'al-Mughira, 968 [d'après S. MAKARIOU, "The al-Mughira Pyxis and Spanish Ivories” A. BORRUT et P. COBB (Eds.), Umayyad Legacies, Leiden, 2010].

sont jamais représentés à cheval). Le Calendrier de Cordoue écrit par 'Arib b. Sa'ad al-Kâtib en 961 pour le calife al-Hakâm (961-976) peut fournir quelques éclairages. En effet, il décrit ainsi le mois de janvier: "c'est un naw' favorable. Le lait et les dattes abondent chez les Arabes durant cette période. La pluie qui tombe alors est appelée rabi' (pluie fertilisante)" ${ }^{35}$. On pourrait à la lumière de ce texte considérer le médaillon à la fois comme une allusion au mois de janvier et aux pluies fertilisantes de ce mois. Il est d'ailleurs pas inutile de souligner ici que le terme employé pour désigner les pluies de ce mois "rabi" désigne aussi la saison du printemps et sert fréquemment de métaphore pour décrire la générosité des califes omeyyades de Syrie. Une autre scène de cueillette figure sur la boîte de Sayf al-Dawla où deux figures en pied semblent cueillir les fruits d'un arbre au tronc tressé. Il est d'ailleurs difficile de déterminer si les personnages cueillent des fruits ou s'ils sont occupés à poliniser, greffer ou tailler l'arbre: autant de pratiques agricoles évoquées dans le Calendrier de Cordoue.

b) Les représentations de vendangeurs: ce sujet est représenté sur une boîte anonyme datée du XIe siècle. On y voit au milieu d'un décor végétal foisonnant deux figures vêtues de tuniques courtes et pieds nus, donnant l'impression de danser en

35 R. DOZY, Le calendrier de Cordoue, Leiden, 1961, pp. 34. 
se tenant par la main. Ces caractéristiques sont celles que l'on retrouve sur la plupart des scènes de vendanges qui servent à représenter le mois de septembre dans les calendriers de l'Antiquité tardive et du Moyen Âge. A titre d'exemple, on peut citer le fameux calendrier en mosaïques qui décorait une riche villa de l'Afrique proconsulaire (al-Djem, IIIe siècle) ${ }^{36}$. On peut aussi évoquer les scènes de vendanges représentées dans les mosaïques romaines de Cherchel (Algérie, IVe siècle) ${ }^{37}$ ou au Proche-Orient, celles qui se trouvent dans le décor de l'église de Saint Christophe à Qabr Hiram (Liban, VIe siècle) ${ }^{38}$ ou de 1'église des Saints Lot et Procope à Khirbat al-Mukhayyat ${ }^{39}$.

c) Les images de fauconniers et "le cycle des faucons". L'image des fauconniers apparaît sur plusieurs ivoires et en particulier sur la pyxide d'al-Mughira. Ce sujet est bien évidemment à mettre en rapport avec l'un des passe-temps favori des princes omeyyades de Syrie et d'Espagne. On le retrouve d'ailleurs sur un des reliefs récemment mis au jour à Qasr al-Hayr al-Gharbî ${ }^{40}$, mais aussi dans le calendrier de Qusayr 'Amra. L'introduction de fauconnier dans l'iconographie des calendriers s'observe d'ailleurs avant l'Islam sur plusieurs calendriers de l'Antiquité tardive (Argos, VIe siècle $)^{41}$ et surtout sur le calendrier qui décore l'église de Saint Elias, Marie et Soreg à Jerash. La pyxide de Mughira offre une autre occurrence du thème des faucons (fig.5). La scène montre trois nids de faucons: le nid central avec un faucon et trois de ses oisillons et deux autres nids de part et d'autre où l'on peut voir les faucons femelles couvant leurs œufs alors que deux hommes tendent leurs mains vers les nids. Cette scène inhabituelle n'est pas sans rappeler de nombreux passages du Calendrier de Cordoue où l'auteur évoque à plusieurs reprises le cycle des faucons qui s'étend sur plusieurs mois de janvier à mai. On y lit par exemple pour le mois de janvier "les oiseaux s'accouplent; les faucons de l'espèce valencienne restent dans leurs nids et commencent à cocher" "42; pour le mois de mars: "c'est le mois où les femelles faucons de l'espèce valencienne pondent leurs oufs et les couvent trente jours" ${ }^{43}$; pour le mois d'avril "les petits de l'espèce valencienne sortent des oufs puis se couvrent de plumes" 44 ; pour le mois de mai: "les faucons muent et leur mue dure jusqu'au début du mois d'aout'. L'éclairage offert par le Calendrier de Cordoue plaide en faveur de l'hypothèse d'une scène calendricale, voire une possible allusion au cycle ordonné de la nature garanti par le califat.

Les différences iconographiques et morphologiques entre les calendriers monumentaux des Omeyyades et les quelques sujets calendricaux de ces objets andalous sont nombreuses (échelle, technique, calendrier complet et représentations allégoriques en Syrie/allusions à des thèmes calendricaux et scènes plus rustiques sur les ivoires).

\footnotetext{
36 K. DUNBABIN, Mosaics of the Greek and Roman World, Cambridge, 1999, fig. 113.

37 Ibidem, op.cit., 1978, pl. 105.

38 A. REINACH, Répertoire des peintures grecques et romaines, Paris, 1922, pp. 352-353.

39 M. PICIRILLO, op.cit., 1993.

40 D. GENEQUAND, op.cit., 2010b, p. 82, fig. 130.

41 G. AKERSTROM, op.cit., 1974, fig. 12 et 13.

42 R. DOZY, op.cit, 1961, p. 36.

43 Ibidem, p. 60.

44 Ibidem, p. 76.
} 


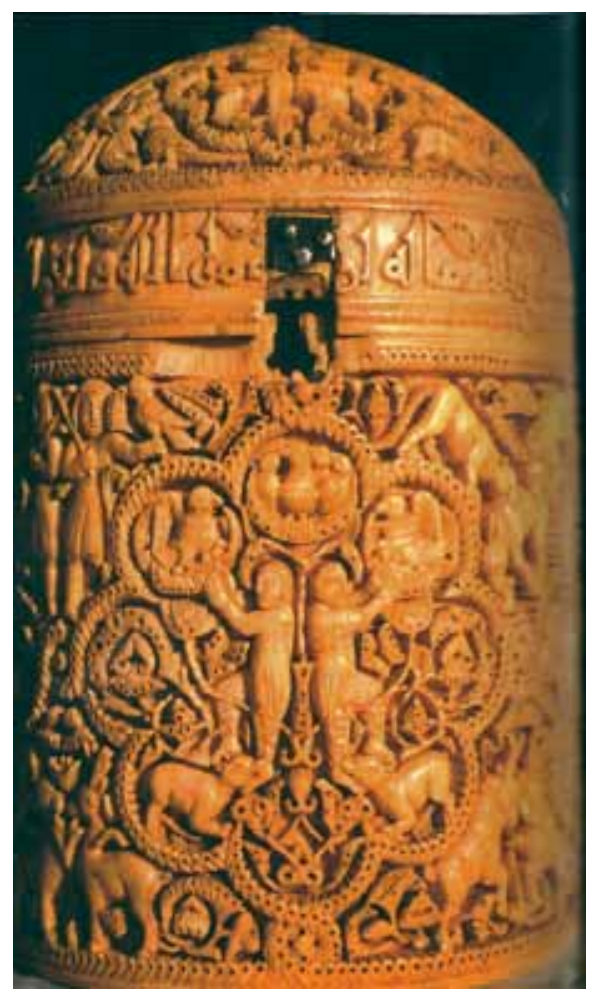

Fig. 5. Pyxide d'al-Mughira, 968 [d'après S. MAKARIOU, "The al-Mughira Pyxis and Spanish Ivories” A. BORRUT et P. COBB (Eds.), Umayyad Legacies, Leiden, 2010].

Pourtant, la fonction de ces thèmes dans la propagande visuelle des Omeyyades de Syrie et d'Espagne semble comparable. D'ailleurs remarquons que la plupart des figures trônantes sur les ivoires portent des attributs végétaux (grappe de raisin, fleur). Ces scènes de trône apparaissent comme des versions très abrégées des programmes des Omeyyades de Syrie et en particulier du programme de Qusayr 'Amra dont la travée centrale représente, je le rappelle, un prince trônant sur l'eau surplombé d'un décor de vigne et flanqué des Saisons, de Ge et d'un calendrier. Une dernière analogie mérite d'être soulignée: la fréquente combinaison entre scènes de chasse et thèmes agricoles qu'on retrouve dans les programmes des résidences Omeyyades de Syrie, mais aussi sur la boîte de Pampelune et celle d'al-Mughira. Ces combinaisons pourraient refléter l'association évoquée dans la poésie arabe classique entre le succès de la chasse et la venue d'une pluie abondante (Imru'l Qais, 'Umar ibn Abi Rabi'a, plusieurs inscriptions sudarabiques). Les inscriptions formulaïques des ivoires andalous pourraient aussi s'inscrire dans cette perspective de lecture dans la mesure où le vocabulaire utilisé est très étroitement lié à cette notion d'abondance agricole et 
de Felicitas (barakât, rahmât, ni'mât, surûr). Ces éléments suggèrent d'une part une certaine forme de continuité dans les représentations du pouvoir omeyyade puisque dans les deux cas, c'est l'image d'un pouvoir nourricier, garant de la fertilité et d'un cycle naturel ordonné qui semble être projetée; et d'autre part la possibilité d'un langage partagé, d'une koiné de la représentation du pouvoir au sein du vieux monde connecté (la Mediterranée).

\section{DISCUSSION: ÉCHO LOINTAIN DES OMEYYADES DE SYRIE ET/OU FRUIT DE LA RIVALITÉ MIMÉTIQUE QUI OPPOSE LES POUVOIRS POLITIQUES CONTEMPORAINS?}

Pour expliquer la présence de thèmes calendricaux et agricoles sur les ivoires d'alAndalous, plusieurs facteurs doivent être invoqués: des pratiques proprement artistiques et des modes iconographiques partagées dans les régions du bassin méditerranéen, des cadres référentiels spécifiques à l'Andalus comme l'essor de la littérature agronomique ou celle des "calendriers", le contexte économique et la révolution agraire. Mais il est aussi possible d'envisager cette iconographie comme le produit de deux autres forces: l'une orientée vers le passé des Omeyyades de Syrie, l'autre vers les califats rivaux des Abbassides et Fatimides.

\subsection{L'HYPOTHÈSE D'UNE FORME DE CONTINUITÉ DANS LA MISE EN SCÈNE DU POUVOIR OMEYYADE}

Peut-on considérer le discours visuel des ivoires andalous comme un écho lointain de la propagande visuelle des Omeyyades du Bilâd al-Shâm? Ce parallélisme est tentant d'autant que plusieurs travaux ont mis en lumière les nombreuses affinités entre l'architecture palatiale de Madinat al-Zahra et celle des Omeyyades de Syrie ${ }^{45}$. Pourtant l'hypothèse d'un transfert de formes de la Syrie à l'Espagne se heurte dans notre cas à des difficultés substantielles. La notion d'influence a tendance à évacuer la réflexion sur les modalités de diffusion. Quel aurait été le mode de diffusion de cette mémoire ou de ce souvenir de l'iconographie syrienne en al-Andalus? S. Calvo Capilla a très bien montré le rôle fondamental joué par les historiens et les textes dans la construction de la filiation entre les Mosquées de Damas et Cordoue ${ }^{46}$. Or, dans le cas de "l'art privé" des Omeyyades de Syrie, l'absence de textes rend très improbable la possibilité d'une influence ou d'une filiation impulsée par les historiens arabes. A ce sujet, Denis Genequand parle d'ailleurs du caractère "éphémère" de l'architecture palatiale syrienne qui n'a pas laissé de témoignage écrit ${ }^{47}$. Dans la formation de cette iconographie andalouse, la possibilité d'une influence au niveau

\footnotetext{
45 A. ALMAGRO, "Los Palacios Omeyas, entre Oriente y Occidente", A. BORRUT et P. COBB (Eds), Umayyad Legacies, pp. 475-513.

46 S. CALVO, "Analogies entre les grandes mosquées de Damas et Cordoue: mythe ou réalité", A. BORRUT et P. COBB (Eds.), Umayyad Legacies, pp. 281-311.

47 D. GENEQUAND, op.cit., 2010a, pp. 444-445.
} 
des conceptions du pouvoir me paraît plus vraisemblable qu'un transfert de formes artistiques du Proche-Orient vers l'Andalus. L'association entre les Omeyyades de Syrie et l'eau ou l'abondance agricole prend une place trop particulière dans l'iconographie des châteaux du désert, les sources épigraphiques et textuelles pour ne relever que du hasard. Il ne serait donc pas étonnant que ses descendants andalous, aient eux-mêmes repris l'eau et l'agriculture comme faire-valoir de leur art de gouverner, come argument de leur légitimité califale. A cet égard, Une étude des nombreuses inscriptions arabes d'Espagne pourrait s'avérer très instructive. Trouve-t-on, par exemple, des inscriptions sur des ouvrages hydrauliques (barrages, citernes) qui témoigneraient comme dans le cas syrien de l'implication réelle, fictive ou mise en scène du pouvoir andalou dans la gestion de l'eau et de l'agriculture? Il serait également utile de traquer dans les sources textuelles andalouses toutes les références à cette fonction nourricière du pouvoir et déterminer si une filiation est établie ou pas avec les Omeyyades de Syrie sur ce point précis. "La littérature califale" pourrait être mise à contribution: Le Fisal d'Ibn Hazm, la Chronique anonyme de'Abd al Rahman III, le Livre des juges de Khushani, le Muqtabas d'Ibn Hayyan ou les Annales de 'Isa al-Razi, al-Razi, la Risala fi-l Qada wa'l hisbba d'Ibn 'Abdun. Comme l'a noté G. Martinez-Gros, "il est vrai que les lettrés d'al-Andalus se sont peu intéressés aux réalités 'économiques', mais ils n'ignorent pas que la prospérité du royaume, le bien des musulmans et la force du califat dépendent de la régularité des pluies, des saisons et de la productivité agricole". A titre d'exemple, on peut évoquer les quelques passages des Annales d'al-Râzî qui traitent du bienfait des pluies sur les récoltes ou de la grande crainte des sécheresses. Ibn 'Abdun dans son traité du XII ${ }^{\mathrm{e}}$ siècle, après avoir défini le prince comme "centre de la société" insiste sur les encouragements qu'il doit prodiguer à l'agriculture: "Le prince a pour mission d'assurer la survie de la communauté en écartant la pauvreté et la disette qui forcent les hommes à s'expatrier". Il écrit: "l'agriculture est la base de la civilisation. C'est d'elle que dépend la vie entière. Pour du grain, on voit se perdre existence et richesses, pour lui, des villes et des hommes changent de maître"48.

\subsection{LE RÔLE DES DYNAMIQUES INTERRÉGIONALES ET DES EFFETS DE LA CONCURRENCE AVEC LES ABBASSIDES ET LES FATIMIDES: UN CAS DE RIVALITÉ MIMÉTIQUE?}

Les hypothèses d'une émulation de lointains modèles omeyyades dans la mise en scène du pouvoir andalous ou celle d'un langage visuel partagé au sein du bassin méditerranéen ne doivent pas exclure d'autres facteurs. Cette koiné visuelle au sein du monde méditerranéen n'est-elle pas induite par la compétition que se livrent les pouvoirs politiques contemporains? D'un côté, les effets de la concurrence et des rivalités mimétiques on pu jouer entre les différentes conceptions impériales de Byzance, de l'empire d'Occident et d'Islam. On sait par exemple grâce à une ekphraseis que l'empereur byzantin Leo VI (886-912) avait fait décorer un bain du palais de

48 G. MARTINEZ GROS, L'idéologie omeyyade, Madrid, 1992, pp. 134-135; p. 314. 
Constantinople par des représentations le mettant en scène avec des allégories associées à la fertilité et l'abondance (Gê portant des fruits) ${ }^{49}$. D'un autre côté, ces rivalités ont pu aussi s'exercer entre les différentes conceptions califales (califat abbasside, omeyyade d'al-Andalus et fatimide). Certains aspects de l'iconographie des Abbassides et des Fatimides mériteraient à ce titre d'être réexaminés. Par exemple, la représentation d'une figure portant un agneau sur l'une des bouteilles découvertes à Samarra ${ }^{50}$ présente des affinités avec le type iconographique traditionnellement utilisé pour la personnification du mois d'Avril dans plusieurs calendriers (Argos, Beisan II, Awzai, Marciana Cod. gr 540). Cela suggère l'hypothèse d'un recours l'iconographie calendricale dans l'art abbasside. De plus, la représentation de cornes d'abondance dans le décor du palais de Jawsaq al-Khaqani semble participer d'une même volonté d'insister sur le caractère nourricier du pouvoir. Du côté des Fatimides, il n'est pas inutile de remarquer que certains thèmes agricoles (vendanges, récoltes) sont insérés dans des cycles princiers comme on peut l'observer sur certaines plaques de revêtement en ivoire ${ }^{51}$.

Dans les fondements de la légitimité des pouvoirs, on évoque souvent les arguments dynastiques ou théologiques, mais la capacité à garantir l'abondance à ses sujets a sûrement été un enjeu dans la compétition qui a opposé les trois califats. Reste un problème de méthode dans l'interprétation proposée ici et que l'on peut résumer en une question: un degré de généralité trop grand ne risque-t-il pas d'aboutir à écraser les spécificités contextuelles sous des catégories qui fonctionnent partout et toujours comme c'est le cas du "pouvoir nourricier" et de sa mise en image?

49 P. MAGDALINO, "The Bath of Leo the Wise and the Macedonian Renaissance Revisited: Topography, Iconography, Ceremonial, Ideology”, Dumbarton Oaks Papers, 42 (1998), pp. 97-118.

50 D. S. RICE, "Deacon or Drink: Some Paintings from Samarra Reexamined”, Arabica, 5 (1958), pl. IV, A.

51 Egypte, XII ${ }^{\mathrm{e}}$ siècle, plaque conservée au Museum fur Islamische Kunst, Berlin, I 6375. 